\title{
Condiciones laborales de profesores egresados del programa de postítulo de mención en matemática para segundo ciclo básico*
}

\author{
Working conditions of teachers who graduated from a Subject-Specific \\ Graduate Program of Math for Middle School
}

\author{
Christian Miranda Jaña ${ }^{a}$, Claudia García Yáñez $z^{a}$ Ricardo Abarca Alarcón ${ }^{a}$, \\ José Manuel Medina Andrade ${ }^{b}$, Sebastián Molina Aguilara ${ }^{a}$, José Luis Medina Moya ${ }^{c}$ \\ ${ }^{a}$ Universidad de Chile. \\ christian.miranda@u.uchile.cl, cgarciayan@gmail.com,rabarca@uchile.cl, sebamolina@ug.uchile.cl \\ ${ }^{b}$ Universidad de Las Américas, Chile. \\ jmedina@udla.cl \\ ${ }^{c}$ Universidad de Barcelona, España. \\ jlmedina@ub.edu
}

\begin{abstract}
RESUMEN
Las condiciones laborales y la formación permanente son factores relevantes en el desarrollo profesional docente. Este estudio busca describir el cambio en las condiciones laborales de docentes formados en el Programa de postítulo de mención en matemática para segundo ciclo básico luego de tres años de su egreso, desde la perspectiva del desarrollo profesional sistémico. El método utilizado es cuantitativo y considera la aplicación de un cuestionario a una muestra de 189 docentes, utilizado para el análisis de los datos estadística descriptiva. Los resultados muestran que las condiciones laborales cambian de modo positivo, siendo de mayor variación las de tipo pedagógicas y menor las de tipo contractuales e institucionales. Estos hallazgos discuten la pertinencia de considerar las condiciones laborales del profesorado en las políticas sectoriales y en los estudios sobre el desarrollo profesional docente.
\end{abstract}

Palabras clave: formación docente, formación permanente de docentes, condiciones de empleo del docente, método cuantitativo.

\begin{abstract}
Working conditions and the quality of training programs have a great influence on teacher professional development. This study aims to describe any changes in the working conditions of teachers who graduated from the Subject-Specific Graduate Program of Math (PPMat, for its Spanish acronym), three years after graduating. In this quantitative study, a questionnaire applied to 189 teachers and descriptive statistics were used. The results show that working conditions change positively: higher variations were found in pedagogical and lower variations were found in contractual conditions and institutional conditions. These findings discuss the importance of considering teacher labor conditions in educational policy and in teacher professional development studies.

Key words: Teacher training, In-service teacher training, Teachers conditions of employment, quantitative method.
\end{abstract}

\footnotetext{
* El artículo se desarrolla en el marco del Núcleo de Investigación y Formación Docente de la Universidad de Chile, se adscribe a los Fondecyt Nº1181772 y 1211417 y cuenta con el apoyo de FACSO a través del Proyecto FPCI 04-2017.
} 


\section{INTRODUCCIÓN}

Las condiciones laborales y la formación permanente son factores relevantes en el debate científico internacional referido a cómo mejorar la atracción, retención y el desarrollo profesional del profesorado (Goldhaber, Krieg y Theobald, 2016; Sutcher, DarlingHammond y Carver-Thomas, 2016; Fern, 2017; Geiger y Pivovarova, 2018). Asimismo, tales factores forman parte del análisis sistémico del desarrollo profesional (Monarca y Manso, 2015) y representan aspectos clave en la toma de decisión del profesorado respecto de su movilidad laboral (Ladd, 2018) y al deseo de actualizar sus saberes durante el ejercicio docente para tener más oportunidades en el campo profesional (Ávalos y Valenzuela, 2016).

Por su parte, el profesorado percibe un nivel razonable de satisfacción con la profesión y una actitud positiva hacia la formación permanente, asistiendo de manera recurrente a cursos y talleres (OCDE, 2018). No obstante, reportan insatisfacción respecto de las condiciones de empleo propicias para transferir la formación recibida por factores institucionales como las rutinas de control burocrático escolar (UNESCO-OREALC, 2012).

Lankford, Loeb y Wyckoff (2002) estudian las condiciones laborales del profesorado y su relación con los atributos de las escuelas en el Estado de Nueva York, tales como selectividad, certificaciones, experiencia, desempeño en pruebas estandarizadas, entre otras. En sus resultados se destacan las diferencias en la distribución de las características del profesorado entre escuelas, mostrando variaciones entre establecimientos urbanos y rurales.

Puga, Polanco y Corvalán (2015) analizan las relaciones entre el origen formativo del profesorado con el tipo de dependencia de los establecimientos y las condiciones laborales donde trabajan, destacando que los aprendizajes del estudiantado son influenciados por la formación del profesorado y las condiciones laborales en que estos trabajan.

Según reporta OCDE (2019) las condiciones laborales del profesorado de primaria en Chile se encuentran entre las más descendidas de sus países miembros. Expresión de estas son: a) Tamaño de los cursos, es el más grande entre países que promedian 21 a 23 estudiantes por sala; b) Horas lectivas, ubicándose entre las más numerosas de los países encuestados; c) Salario, el cual es transversalmente bajo en todo el sistema educativo. Sobre esto último, el magisterio percibe en promedio un $16 \%$ del salario menor al de otros trabajadores con igual educación superior (OCDE, 2016 y 2017).

A nivel nacional, los estudios han avanzado en la caracterización de las condiciones laborales del profesorado con especialización en su historia (Troncoso, 2003), según género (Rivera y Miranda, 2009) y trayectoria profesional (González, 2020). Finalmente, Beyer, Miranda y Arancibia (2018) en su estudio sobre docente de segundo ciclo de primaria muestran que según las concepciones del profesorado entrevistado el impacto educativo de la formación recibida estaría mediada por las condiciones laborales previas y posteriores a la especialización.

Pese al avance en las investigaciones sobre el desarrollo profesional docente y la relevancia de las condiciones laborales y la formación recibida en estos temas, no se reportan estudios que de manera directa indaguen respecto de la variación en el tiempo de las condiciones laborales del profesorado luego de la especialización profesional. Así el objetivo de este trabajo es describir el cambio en las condiciones laborales del profesorado especializado para segundo ciclo básico del país, luego de tres años de egreso. 


\section{MARCO CONCEPTUAL DE LA FORMACIÓN Y CONDICIONES LABORALES DEL PROFESORADO}

El proceso de formarse como docente requiere de aprendizajes que solo pueden ocurrir durante el ejercicio mismo de la profesión, por ello, es ineludible la formación permanente de la enseñanza (Ingersoll y Strong, 2011). Esto conlleva entender la formación docente como un proceso continuo, dinámico y de alta complejidad (Ávalos y Matus, 2010).

En Chile, la formación permanente ha sido un componente central de las reformas educativas contemporáneas. En efecto, el nuevo Sistema de Desarrollo Profesional Docente (MINEDUC, 2017) la asume como un derecho y como tema prioritario en las políticas, modelos y tendencias orientadas a mejorar el estatus profesional de la docencia. No obstante, Alvarado (2012) señala que pese al unánime respaldo público a la formación permanente, conceptualmente suele considerarse sinónimo de capacitación, reciclaje, formación permanente, entre otras, donde cada aseveración alude a una concepción político-ideológica. En este estudio se prefiere manejar el primer término, pues es utilizado por el MINEDUC, siguiendo indicaciones propias de la UNESCO.

En ese marco se espera que la formación permanente posibilite la implementación a nivel de aula de políticas sectoriales mediante la especialización profesional, donde las instituciones escolares tienen que viabilizar condiciones laborales adecuadas tanto para la concurrencia docente como para la implementación didáctica y sostenibilidad en el tiempo de las mejoras que la preparación provea. Al respecto, estudios recientes han avanzado en el análisis conceptual de cómo es concebida la formación permanente por parte de investigadores (Quintero, Miranda \& Rivera 2018) y docentes beneficiarios (Améstica, Miranda, Abarca, Medina \& Pinto, 2018), pero poco se ha avanzado en una visión teórica que relacione tales concepciones con las condiciones laborales de los docentes previas y posteriores del perfeccionamiento realizado. Al respecto, Mourshed, Krawitz y Dorm (2015) sostienen que las perspectivas de formación permanente deben tender puentes conceptuales y operativos entre las ideas y condiciones de la formación, la implementación de innovaciones y las evaluaciones de sistemas educativos en progreso como el chileno.

Para los fines de este trabajo se entiende la formación permanente del profesorado como un proceso de aprendizaje profesional de carácter político e ideológico, encaminado al desarrollo integral de sus capacidades y que apela al protagonismo del docente en la continua ampliación de sus experiencias, saberes y actitudes base de la enseñanza.

Los docentes acuden a los programas de formación permanente con una serie de expectativas, orientadas a satisfacer sus necesidades, e intentar procesos de cambio con base en la especialización profesional (Salinas, 2010). Asimismo, los programas de formación representan una respuesta a la falta o insuficiencia de tiempos dentro de la jornada laboral y de estímulos adecuados para la participación en procesos de aprendizaje profesional, tanto a nivel individual como colectivo (Terigi, 2012). Al respecto Blanco (2013) señala que existe una percepción generalizada respecto que los tiempos disponibles dentro de la jornada escolar son insuficientes para la realización de actividades de formación, en sus diferentes modalidades. Se suma a ello, la preocupación por las condiciones contractuales y relaciones laborales del profesorado de primaria y las complejidades pedagógicas y didácticas que su labor integradora demandan (Beca, 2006; Barría, Miranda, Toledo \& Gajardo, 2017). 
Los Programas de Postítulo con Mención buscan responder a tales expectativas, preocupaciones y complejidades. En efecto, su propósito es fortalecer las competencias disciplinares y pedagógicas del profesorado que enseñan en áreas curriculares de alto impacto como matemática (en adelante PPMat). Esto ha llevado a que hasta el 2017 se haya experimentado un incremento significativo en su cobertura, focalización (más del $50 \%$ corresponde a programas para segundo ciclo y en el subsector de matemática), costo y amplitud geográfica, como consecuencia de los procesos de reforma educativa (Beyer, Miranda y Arancibia, 2018).

Por otro lado, siguiendo a Martínez (2001) las condiciones de empleo o laborales se asumen como una categoría de análisis que posibilita relacionar el trabajo del profesorado en la escuela y la formación permanente recibida. Desde esta perspectiva, se alude al carácter multidimensional de la labor docente y a la necesaria consideración de aspectos sociales, políticos, culturales y educativos situados históricamente, que delimitan el marco estructural en el que trabajan y se desarrollan profesionalmente. A los fines de este estudio, la noción de condiciones laborales hace referencia a un conjunto de recursos institucionales, contractuales y pedagógicos que permiten/dificultan la realización del trabajo docente y que aluden a las relaciones de empleo, entre las que se destacan el tipo de dependencia de las escuelas, las modalidades de contratación, el número de estudiantes, la dedicación a la enseñanza de la disciplina de especialización, entre otras.

Desde la perspectiva teórica del desarrollo profesional docente sistémico (Monarca y Manso, 2015) se parte del supuesto que el dispositivo "formación permanente" (operacionalizado en el PPMat) está relacionado a las "condiciones laborales" del profesorado, por lo cual caracterizar sus variaciones empíricamente, permite obtener un marco interpretativo de los cambios y continuidades entre el ingreso y el egreso, posibilitando un análisis integral que complejiza tanto el dispositivo reseñado como el estudio de programas como el PPMat que buscan ejecutar políticas públicas en ámbitos focalizados del desarrollo docente como la especialización profesional.

De este modo, la hipótesis de trabajo es que un análisis de las relaciones propuestas en el modelo teórico evidenciará cambios positivos en las condiciones institucionales, contractuales y pedagógicas del profesorado, luego de tres años de egresado del PPMat.

\section{MÉTODO}

Se opta por un estudio de tipo descriptivo no experimental que articula procedimientos cuantitativos mediante un diseño transversal, con un solo grupo y aplicación de un cuestionario. La investigación se desarrolla considerando desde Stake (2006) variables de entrada (edad, género y años de experiencia), de proceso (formación permanente operacionalizada a través del PPMat) y de resultado (condiciones laborales de tipo institucional, contractual y pedagógicas).

La población del estudio está constituida por docentes en servicio, que laboran en escuelas con subvención pública de todo el país. La proveniencia geográfica se sustenta en el carácter nacional del PPMat. Esto significa que la muestra es heterogénea con relación a los niveles altos y bajos de selección en las variables consideradas y en relación a la diversidad sociocultural que caracteriza al profesorado nacional. En efecto, el sistema educativo chileno se distingue por agrupar los colegios municipales y subvencionados, 
que reciben subvención del Estado y los particulares que se financian con el pago de las familias. Según la Agencia de Calidad (2016), los dos primeros, considerados en el estudio, representan el $92 \%$ de la matrícula nacional.

Se consideró la necesidad de contar con muestras heterogéneas, a fin de aumentar la confiabilidad del instrumental utilizado. El tamaño muestral consideró el procedimiento de muestreo por conveniencia considerando como criterios de inclusión ser docentes egresados de los PPMat (cohortes 2012 a 2015) que al momento del estudio estén trabajando en escuelas municipales y particulares subvencionadas del país y aceptar voluntariamente formar parte de la investigación, aspecto consignado en consentimientos informados. De esta forma, para la selección de la muestra se consideró la fracción de muestreo, en este caso, 189 docentes que representan el $26 \%$ de la población egresada $(\mathrm{N}=539)$ en las cohortes consideradas. Se estima, siguiendo a Catena, Ramos y Trujillo (2003) que esta muestra es suficiente para caracterizar las variaciones en las condiciones laborales de la población estudiada.

La elección del nivel, subsector de enseñanza y programa, tiene por fundamento la discusión internacional sobre cómo mejorar la enseñanza de matemática en el nivel primario (OCDE, 2018), la desaceleración del aprendizaje detectado en este ciclo escolar (MIDE UC, 2009), los estudios previos del PPMat (Miranda, Rivera, Arancibia y Osses, 2012; Ralph, Miranda y García, 2019) y la relevancia de la especialización disciplinar en la Ley 20.903 sobre desarrollo profesional docente (MINEDUC, 2017). También se considera la selectividad y diversidad de las universidades que desarrollan los PPMat (Estatal, Tradicional Privada, Confesional y Privada Laica), todas ellas del Consejo de Rectores y situadas en las regiones económicamente más importantes del país. La Tabla 1 a continuación presenta la distribución final del estudio.

Tabla 1. Distribución de la muestra del estudio según grupos

\begin{tabular}{|c|cc|c|}
\hline Grupos & \multicolumn{2}{|c|}{ PPMat - Región } & Muestra \\
\hline 1 & Universidad Católica de Valparaíso - V Región & 36 \\
\hline 2 & Universidad de Santiago - XIII Región & 50 \\
\hline 3 & Universidad Diego Portales - XIII Región & 37 \\
\hline 4 & Universidad de Concepción - $\quad$ VIII Región & 30 \\
\hline 5 & Universidad Católica de Temuco - IX Región & 34 \\
\hline Total & & & $\mathrm{n}=189$ \\
\hline
\end{tabular}

Fuente. Elaboración propia.

\subsection{CARACTERIZACIÓN DE LA MUESTRA}

De los datos registrados, se constata que hay una mayor proporción de mujeres (63\%) que de hombres que realizan el PPMat, aspecto consistente con estudios internacionales como OCDE (2019) que muestran que el 67\% de los docentes que enseñan en el país son mujeres. 
Además, el rango de edad promedio predominante de los y las docentes al comenzar el programa es entre 27 a 30 años (26\%), lo que permite deducir que el programa es su primera especialización. La mayoría de los docentes que lo realizan proviene de la Región Metropolitana (41\%) y, en menor medida, de Valparaíso (19\%), Araucanía (18\%), Biobío, (16\%) y O'Higgins (5\%) respectivamente. Este resultado da cuenta del cumplimiento parcial del PPMat en su pretensión de cobertura nacional, aspecto que podría relacionarse a los requisitos que pone el Ministerio de Educación (sólo universidades que cuenten con sus carreras de pedagogía acreditadas) y el costo-beneficio que significa para las instituciones participar de este tipo de programas extensivos en el tiempo y de alta exigencia curricular y dotación docente (Beyer, Miranda y Arancibia, 2018).

En cuanto a la experiencia, la mayor proporción de docentes se tituló de 2 a 5 años antes de comenzar el programa (58\%) y tienen de 2 a 5 años de experiencia en el subsector de matemática (65\%). Esto contrasta con estudios previos, donde, la experiencia profesional de los docentes egresados del PPMat se sitúa en torno a los 10 a 15 años y con más de 10 años enseñando en el subsector (Miranda, Rivera, Arancibia y Osses, 2012). Esto estaría significando que el perfil del profesorado que se especializa en las cohortes estudiadas es predominantemente "noveles" que se encuentran en un proceso de transición desde docente en formación hacia un profesional autónomo (Cisternas, 2016). En esta etapa los y las docentes deben adquirir conocimientos profesionales (Marcelo, 2009) para que complementen su falta de experiencia. De este modo la especialización se relaciona a este anhelo de autonomía y profesionalización. Se pueden sumar a ello, las actuales políticas sectoriales que incentivan el ascenso en la carrera profesional a partir de especializaciones en áreas curriculares de alto impacto como matemática y producto de evaluaciones de desempeño que evidencian competencias pedagógicas y disciplinares por sobre otras habilidades docentes (MINEDUC, 2017).

\subsection{INSTRUMENTO DE RECOGIDA DE DATOS}

El instrumento utilizado fue el Cuestionario de Caracterización Docente, creado para los fines del presente trabajo. La construcción del instrumento se realizó en tres etapas, a saber: determinación de las áreas temáticas mediante la estrategia del muestreo teórico a partir de la revisión de instrumentos en uso revisados y la aplicación de la saturación teórica (Strauss y Corbin, 2002); consulta a expertos que consideró agentes del MINEDUC, investigadores educativos de España y Chile y docentes egresados del PPMat, siendo su selección de tipo intencionada (5 de cada entidad), todos con conocimientos sobre los PPMat. Sus respuestas permitieron pasar de áreas a ejes temáticos, aplicando la técnica de análisis de contenido, estableciendo la etapa de inventario y clasificación, generando tres áreas, esto es: a) Condiciones Institucionales. Considera el tipo de dependencia administrativa de los establecimientos educativos donde trabajan los docentes. Se incluyen establecimientos educativos municipales y particulares subvencionados ${ }^{1}$; b) Condiciones Contractuales. Considera el tipo de contrato (indefinido, contrata u honorarios) y las horas de contrato (extensión de la jornada), y; c) Condiciones Pedagógicas. Consideran aspectos relacionados con la enseñanza como ciclo escolar, número de estudiantes por curso y subsectores de enseñanza.

Se excluyen los docentes que laboran en establecimientos particulares pagados por ser casos aislados. 
El instrumento se estructura en dos momentos. El primero aborda las condiciones institucionales, contractuales y pedagógicas, antes del PPMat y el segundo reitera tales condiciones luego de tres años de egreso, siendo de esta forma plausible contrastar la hipótesis de trabajo.

Con el propósito de determinar la consistencia interna del cuestionario y de cada factor, con los ítemes seleccionados, se realizó un análisis de confiabilidad interna a través del Alfa de Cronbach, siendo este 0,71. De este modo el instrumento consta de 3 dimensiones con un total de 35 reactivos.

El análisis considera criterios de equivalencia inicial (programas) representatividad estadística (docentes), credibilidad cualitativa (caracterización de los y las docentes) y validez instrumental (juicio de expertos).

Los datos son registrados en Excel 2013 y posteriormente ingresados al paquete estadístico SPSS versión 21.0. La forma de análisis fue utilizando técnicas de estadísticas descriptivas. Concretamente, se presentan los cálculos del promedio, en una distribución de medias aritméticas para determinar cuál es la medida de tendencia central, y las medidas de variabilidad, mediante el cálculo de la desviación estándar para medir los intervalos que designan número de unidades en el cuestionario. Estadísticas de cada dimensión para conocer los valores centrales, medidas de variabilidad y las representaciones gráficas para cada variable de entrada. Finalmente, se realizó una triangulación mediante la comparación de datos totales con el marco teórico.

\section{RESULTADOS}

Los resultados se estructuran en razón de los ejes o factores de la variable dependiente, es decir, las condiciones laborales. A continuación, se presentan tablas y gráficos de las condiciones institucionales, disciplinares y pedagógicas.

\subsection{CONDICIONES INSTITUCIONALES}

En cuanto al tipo de dependencia administrativa de los establecimientos donde ejercen los docentes de la muestra, las categorías fueron divididas en tres tipos de establecimientos; municipal, particular subvencionado y particular pagado. Los docentes antes de realizar el programa se distribuían de manera equitativa en los dos primeros tipos, perteneciendo un $52 \%$ a municipal y un $46 \%$ a particular subvencionado, sólo hay registros de un caso de particular pagado. La situación luego del PPMat mantiene la misma tendencia, aumentando la dotación de docentes que ejerce en municipal en un $8 \%$ y una disminución de aquellos que ejercen en particular subvencionado en un $6 \%$.

Un primer análisis de las condiciones laborales de tipo institucional de los docentes de la muestra permite observar, en primer lugar, que un criterio de selección de todos los postulantes a los PPMat es trabajar en escuelas con subvención pública, por lo cual el caso del docente que se adscribe al particular pagado es digno de analizar. Si bien el cuestionario permitía responder a la pregunta por el tipo de escuela en una o más opciones, por la importante inversión que asume el Estado es menester que en el futuro se estudien estos casos. Luego, resulta importante relevar la movilidad laboral evidenciada por un grupo de docentes. En efecto, un $8 \%$ de ellos se mueve desde colegios particulares subvencionados 
a municipales. Estos hallazgos confirman estudios internacionales que constatan que los espacios de desarrollo profesional representan factores relevantes en la toma de decisión de docentes en torno a su movilidad (Hancock \& Scherff, 2010) y nacionales donde se constata mayor movilidad en los establecimientos de tipo privado (López, 2015).

Figura 1. Distribución de los docentes según tipo de dependencia

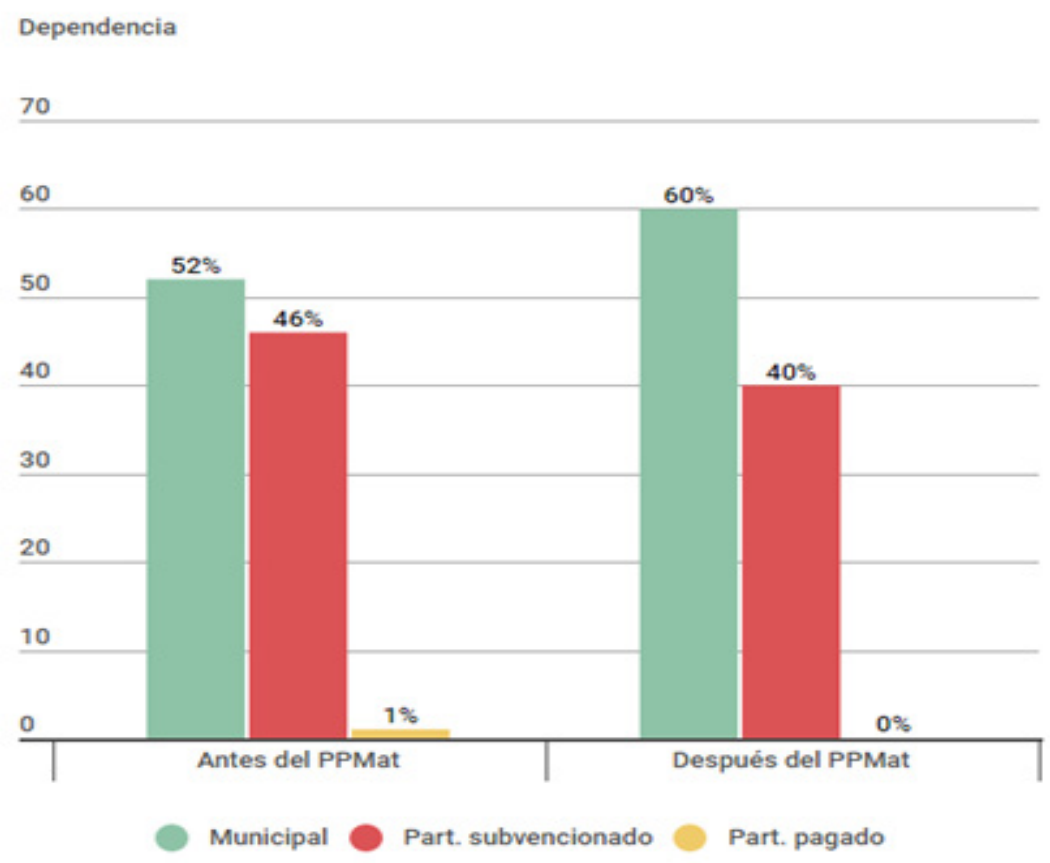

Fuente. Elaboración propia.

\subsection{CONDICIONES CONTRACTUALES}

El tipo de contrato de los docentes se definió en base a tres categorías; indefinido, a contrata y a honorarios. Antes de realizar el PPMat, la tendencia de los profesores se dividía entre dos categorías: indefinido (52\%) y a contrata (46\%), con un bajo grupo de ellos a honorarios (2\%). Luego del PPMat, aumentó el porcentaje de docentes con contrato indefinido un $16 \%$, y disminuyó el grupo a contrata en un $14 \%$ con relación a la primera medición, desapareciendo los casos a honorarios. Estas evidencias se pueden relacionar con los resultados encontrados por Meckes y Bascopé (2010), quienes encontraron que la probabilidad que los docentes sean contratados por colegios aumenta a medida que éstos egresen de instituciones más selectivas, como el caso de las universidades que dictan los PPMat. En ese sentido, la identificación de los cambios en el tipo de contratación de los docentes puede llevar a consideraciones de política formativa. En primer lugar, los 
criterios de selección de los maestros en los programas no contemplan de manera explícita el tipo de contrato que las escuelas tienen con el profesorado, o si las horas de contrato son predominantemente centradas en la enseñanza de la disciplina de especialización antes o después del egreso. Además, los docentes encuestados reportan que en las escuelas municipales los contratos son más estables en comparación con las de particulares subvencionadas. Esto podría implicar que la movilidad hacia las escuelas públicas que garantizan mayor estabilidad laboral puede ser una fuerza impulsora que ponga freno al retiro de los docentes de la profesión, a pesar de que es una suposición común de los gestores escolares y responsables de las políticas (Geiger y Pivovarova, 2018).

Figura 2. Distribución de docentes según tipo de contrato



Fuente. Elaboración propia.

Las horas de contrato de los docentes, fueron definidas según cuatro categorías, a saber; 44 o más, entre 30 y 43, entre 29 y 22 y menos de 22. Antes de realizar el PPMat un $86 \%$ del profesorado se encontraba entre las dos primeras categorías, distribuidos un $62 \%$ en 30 y 43 y un $24 \%$ en 44 o más. Luego de realizar la especialización, un $89 \%$ se mantenía en estas categorías, aumentando la dotación de docentes con 44 o más en un $13 \%$ y disminuyendo el grupo de docentes 30 y 43 en un 10\%. En relación con las otras 
categorías, los y las docentes que tienen entre 22 y 29 horas, bajaron su proporción en un $3 \%$ y tanto antes como después se mantuvieron estables los casos con menos de 22.

Figura 3. Distribución de docentes según horas de contrato

\section{HORAS DE CONTRATO}

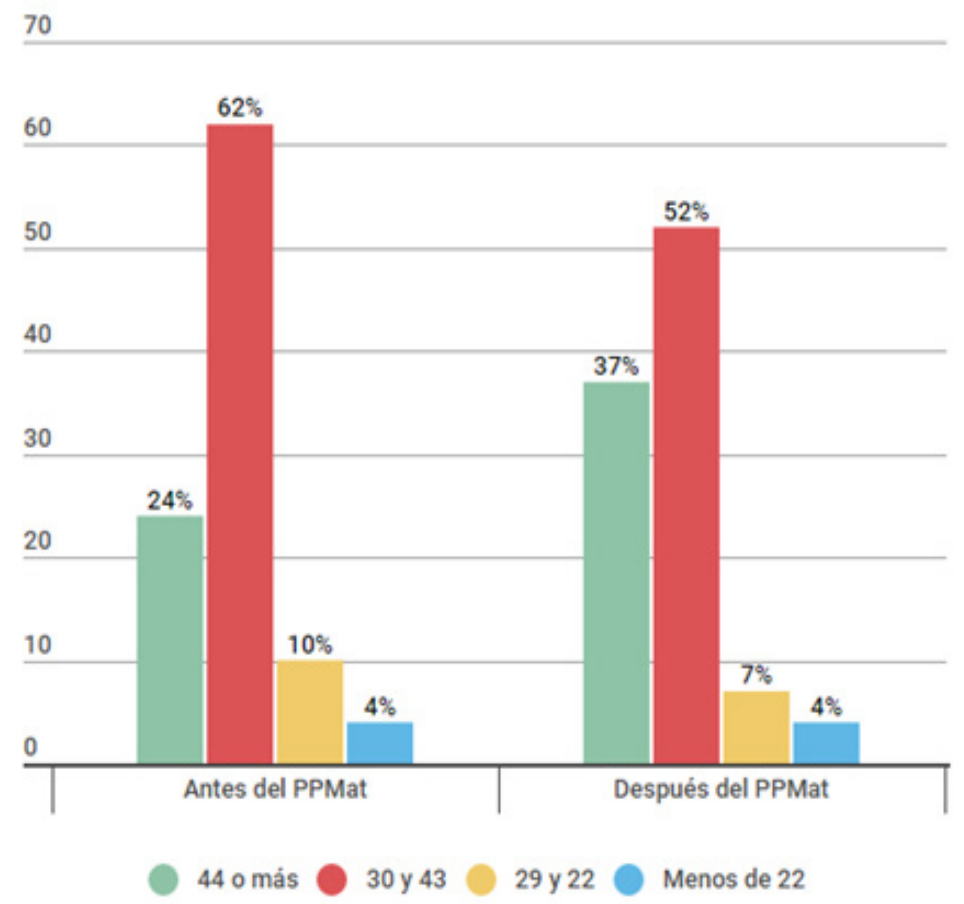

Fuente. Elaboración propia.

La caracterización de las condiciones laborales de tipo contractual del profesorado de la muestra permite observar, en primer lugar, que un grupo importante de docentes cuenta o accede con posterioridad al PPMat a un contrato de empleo de tiempo completo o mayor a 30 horas semanales. Luego, y en consistencia con lo anterior, la variación de horas de contrato del profesorado antes y después del programa se mantuvo en los rangos superiores (entre 44 y 30 horas), donde un grupo importante de ellos (24\%) aumentó la cantidad de horas. A la par con investigaciones internacionales (Ladd, 2018) y nacionales (Ávalos y Valenzuela, 2016) parece ser que como la mayoría de los trabajadores, el gremio docente toma sus decisiones sobre formación, permanencia y movilidad según criterios de selectividad, compensación y calidad del ambiente laboral y tienden a desempeñarse en escuelas donde tales criterios sean mejor percibidos, lo que conduciría a índices de retención y efectividad más altas entre el profesorado. 
Si bien no se puede afirmar que las mejores condiciones contractuales que intenciona la nueva Carrera Docente (Centro de Políticas Públicas, 2012) en las escuelas con subvención pública condujeron al profesorado a mayores índices de ampliación de la carga horaria, parece evidente que existe una relación positiva entre la retención/movilidad docente y las condiciones de trabajo según el tipo de administración de las escuelas.

\subsection{CONDICIONES PEDAGÓGICAS}

La tipificación de los ciclos de enseñanza en el cuestionario se consignó en tres categorías: primer ciclo, segundo ciclo y primer y segundo ciclo. Con respecto al grupo de docentes que se desempeñan exclusivamente en primer ciclo la situación se mantiene estable, representada por un $17 \%$. En cambio se observa una variación notoria en la muestra de docentes que se dedican exclusivamente al segundo ciclo, casi duplicándose luego del egreso, lo cual se expresa en un incremento de un 23\% del total. En proporción similar disminuye la tendencia de docentes con dedicación en primer y segundo ciclo, bajando de manera significativa en un $22,1 \%$.

Lo anterior, significa que un nuevo patrón surgió en las condiciones del profesorado luego del egreso. En efecto, el análisis muestra que la tendencia mayor de la muestra de sujetos era desempeñarse en toda la extensión de la educación primaria -primer y segundo ciclo- en comparación con la situación posterior, donde la mayoría pasa a concentrar su enseñanza en el segundo ciclo, y al mismo tiempo, un grupo menor de docentes pero no por ello menos importante sigue enseñando solo en el primer ciclo. En conjunto, estos tres hallazgos señalan que la formación especializada marcaría diferencias entre el profesorado en sus escuelas, pudiéndose intencionar en las políticas de desarrollo profesional, generando incentivos adicionales para permanecer en tales escuelas y concentrarse en enseñar en el ciclo de especialización. Esto también podría implicar, por ejemplo, que tales docentes puedan ser considerados como mentores de otros docentes nóveles o bien en formadores de sus pares sin especialización que enseñan matemática en otros establecimientos de la misma localidad. 


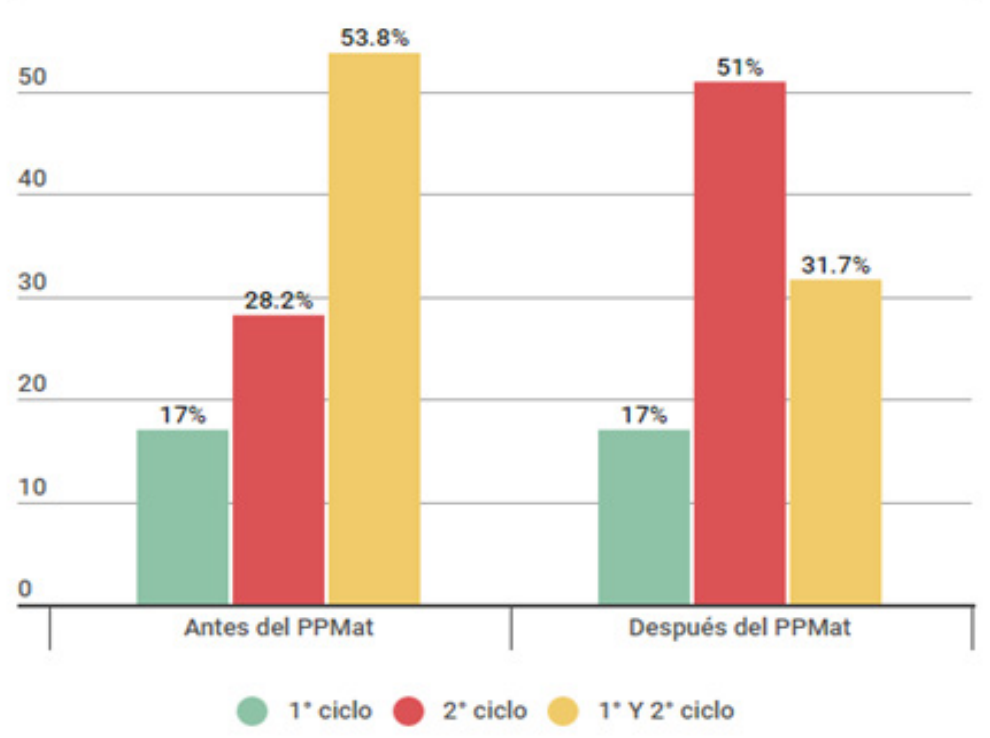

Fuente. Elaboración propia.

Para el número de estudiantes por docente se utilizaron cuatro categorías; más de 40, entre 39 y 30 , entre 29 y 20 y menos de 20 . Entre los resultados se obtuvo que la mayoría del profesorado (37\%) trabajaba con 39 a 30 estudiantes, situación que se vio aumentada en un $11 \%$ luego de la especialización. En cuanto a la categoría de menos de 20, antes del PPMat un $12 \%$ de docentes trabajaba en esta situación y luego de este decrece en un $10 \%$.

Un análisis integrado de este aspecto con los anteriores (ver Tabla 2) permite constatar que cursos numerosos no estarían condicionando el desarrollo profesional de docentes en las escuelas. Al respecto, estudios internacionales (Alain et al., 2013) señalan que es poco probable que el profesorado se retire de las escuelas debido al número de estudiantes por sala. Si bien estos hechos en conjunto no implican que la especialización y el número de estudiantes por sala mantendría a docentes noveles en las escuelas, relevan la importancia las condiciones pedagógicas en las percepciones del profesorado sobre la satisfacción con sus trabajos. 
Figura 5. Distribución de docentes según número de estudiantes

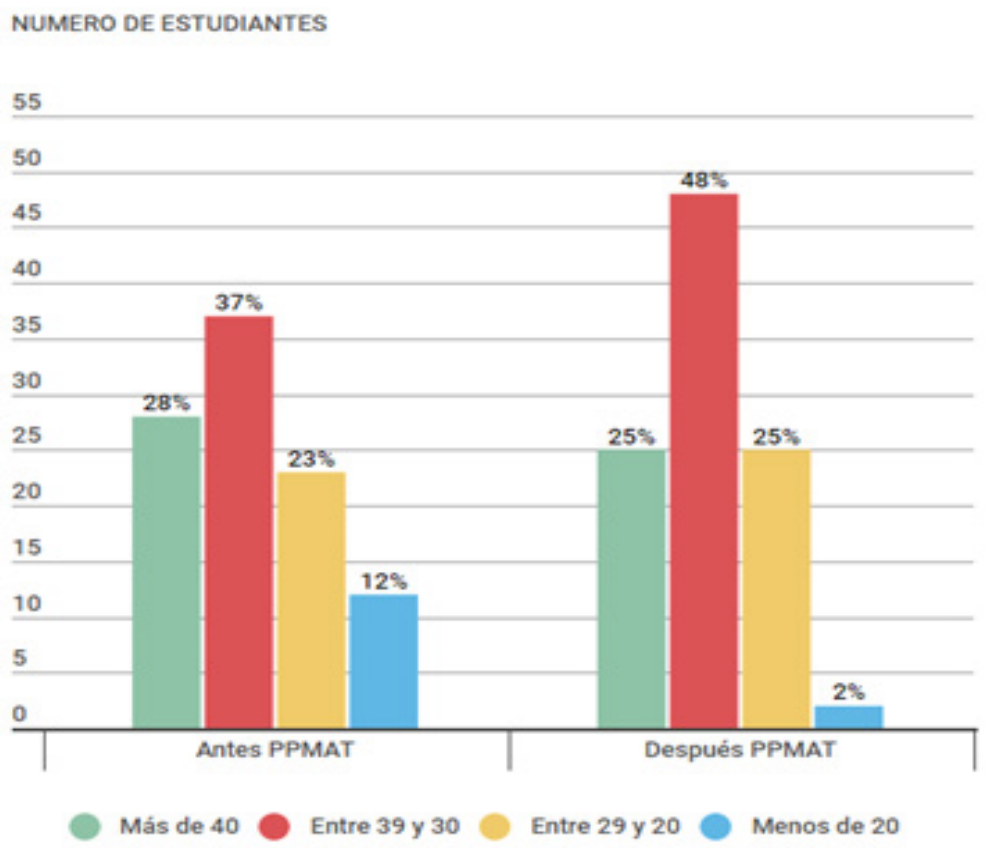

Fuente. Elaboración propia.

Con relación al subsector curricular que imparte el profesorado se consideraron tres categorías; diversos subsectores que no incluyen matemática, matemática y 1 o más subsectores y solo en matemática. Lo observado en esta dimensión indica que el profesorado que no imparte clases de matemática baja en un $6 \%$ luego de cursado el programa. En contraste, aumentan de manera notable (36\%) los que solo enseñan matemática, y el grupo de docentes que se desempeñan en varios subsectores incluido matemática disminuye en un $30 \%$. Estos hallazgos son de alta relevancia, pues proporcionaron antecedentes del resultado de los programas de especialización disciplinar en las condiciones laborales de tipo pedagógicas de los docentes egresados. 
Estudios Pedagógicos XLVII N 3: 7-25, 2021

CONDICIONES LABORALES DE PROFESORES EGRESADOS DEL PROGRAMA DE POSTÍTULO DE MENCIÓN EN

MATEMÁTICA PARA SEGUNDO CICLO BÁSICO

Figura 6. Distribución de docentes según subsector antes del PPMat Subsector en que trabajan docentes antes del PPMat

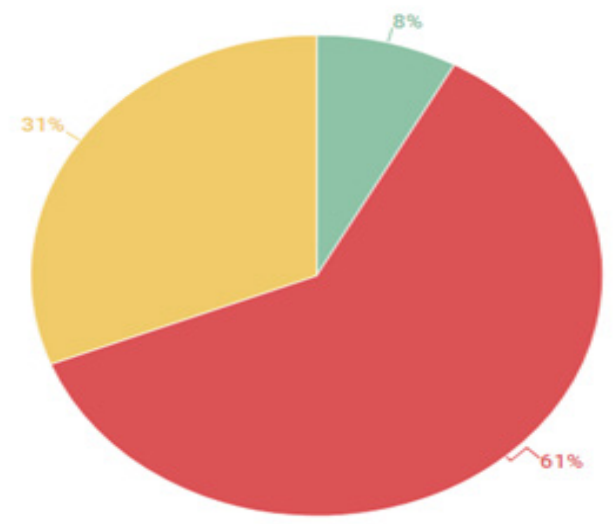

Diversos subsectores s/mat

Mat. y 1 o más Subsect.

Mat.

Fuente. Elaboración propia.

Figura 7. Distribución de docentes según subsector después del PPMat Subsector en que trabajan docentes después del PPMat



Diversos subsectores s/mat

Mat. y 1 o más Subsect.

Mat.

Fuente. Elaboración propia. 
De este modo, la hipótesis del estudio fue contrastada mediante el análisis de medidas de tendencia central y las variaciones que se reportan entre el antes y después del PPMat por parte del profesorado encuestado. Un primer análisis descriptivo del modelo indicó que las características personales no eran un buen indicador de la variable condiciones laborales, dado que presentaba más bien un rasgo estructural del profesorado. Esta consideración permitió obtener un modelo más ajustado de datos. La prueba final, muestra que usando el método de distribución de las medidas de tendencia central, el ajuste del modelo hipotético resultó adecuado. Esto es, se evidencian cambios en las condiciones institucionales, contractuales y pedagógicas del profesorado, luego de tres años de su egreso. Sobre lo anterior, se muestra una variación positiva pero moderada sobre las condiciones institucionales, siendo relevante el cambio de establecimientos particulares subvencionados a los municipales de una parte importante del profesorado especializado. Luego, las condiciones contractuales, muestran una variación positiva en relación al tipo de contrato laboral, donde la tendencia pasa de contrata a indefinido. Finalmente, en las condiciones pedagógicas se constata una variación positiva en el nivel de enseñanza, aumentando la focalización del trabajo en el segundo ciclo básico. Además, en la cantidad de estudiantes que atiende también se observan variaciones importantes, concentrándose en curso de un número alto (30 a 39), producto probablemente del traspaso de un tipo de establecimiento a otro. Por otro lado, la distribución por subsector evidencia un aumento al mostrar una dedicación horaria que pasa de parcial $(31 \%)$ a mayoritaria $(67 \%)$ en la muestra del trabajo. Finalmente, el contraste del modelo destaca el rol del dispositivo formación permanente en el cambio en las condiciones laborales del profesorado, situación expresada conceptualmente en el desarrollo profesional sistémico (Monarca y Manso, 2015) y que puede ser considerado en la evaluación de la carrera docente, planteada por el nuevo sistema de desarrollo profesional (MINEDUC, 2017).

En síntesis, los análisis realizados han mostrado un conjunto de evidencias, patrones y tendencias generales sobre los cambios en las condiciones laborales del profesorado egresado de los PPMat que participaron en el estudio y la discusión que esto puede generar en el marco de los estudios nacionales e internacionales en la materia. Sin perjuicio de lo anterior, no se puede relacionar tales elementos con el impacto de la formación permanente en tales temas y, por lo tanto, no se puede inferir relaciones causales entre el PPMat, las condiciones laborales y la valorización de la especialización profesional. Futuros estudios tendrán que contrastar estos hallazgos, limitándose a docentes egresados de universidades selectivas y que trabajan en instituciones con similares características socioculturales, esto es escuelas municipales y particulares subvencionadas. Otro elemento que considerar es que los datos sobre las condiciones de trabajo se recopilaron antes de la promulgación del nuevo sistema de desarrollo profesional docente, por ende su consideración debe primar en futuros análisis dada su relevancia en la carrera docente actual. Pese a esto último, y dado que el equipo de investigación contaba con estudios previos en la materia y que se aplicó el cuestionario a una muestra representativa de docentes, se puede sostener que los hallazgos proporcionan evidencias que valen para todos los docentes egresados de las cohortes 2012 a 2015. Finalmente, es importante consignar que los resultados de este trabajo no pueden representar una evaluación de las universidades formadoras o del profesorado egresado o que los instrumentos utilizados puedan servir para tales propósitos. 


\section{DISCUSIONES Y CONCLUSIONES}

Los hallazgos de este trabajo son relevantes en el campo conceptual y operativo de los estudios sobre el desarrollo profesional docente sistémico, pues evidencian cómo la ampliación de los conocimientos base de la enseñanza, en este caso de matemática, se relacionan con las condiciones laborales del profesorado egresado de los PPMat.

Las tendencias generales para la muestra encuestada en relación a las condiciones personales del profesorado que ha egresado del PPMat han variado entre las cohortes iniciales y la del estudio. Siendo estas últimas caracterizadas como de una edad promedio de entre 27 y 30 años, en su mayoría de género femenino, poseer título profesional hace menos de 5 años y contar con una experiencia en la enseñanza de matemática entre 2 y 5 años, constando con ello, un perfil de profesor/a en búsqueda de su autonomía, distanciándose del perfil con mayor experiencia, encontrado en estudios previos.

En relación a condiciones laborales del profesorado en términos institucionales, contractuales y pedagógicas se puede concluir que éstas presentan variaciones, siendo las de mayor cambio, las pedagógicas asociadas a subsector de enseñanza y ciclo escolar y menor en las institucionales según dependencia de los establecimientos y contractuales en la categoría tipo de contrato.

Entre las limitaciones del estudio se puede mencionar la muestra, que está determinada por un factor de selección externo (PPMat), a su vez, sujeta al profesorado que acude a instancias de formación permanente, específicamente en al área disciplinaria de interés para los investigadores, en este sentido el sondeo que presentan las conclusiones no pretenden levantar una generalización pero sí identificar una tendencia susceptible de ser profundizada o extrapolada con otros estudios equivalentes o sucesivos que permitan ir construyendo un itinerario de indagación como se plantea en las proyecciones. Otra limitación asociada a la primera, pero de orden más cualitativo, es el foco en un área específica, la matemática en este caso, pero cuya decisión se basa en priorizar una temática considerada de alto impacto por las políticas públicas con la consecuente cobertura que se le ha dado en el tiempo de acuerdo a cifras de cursos y participantes en instancia de desarrollo profesional docente tal como se documenta en el marco referencial, pero que sin duda representan sólo una dimensión de la realidad del magisterio nacional.

Entre las proyecciones de este trabajo se encuentran preguntas relativas a ¿cómo los gestores nacionales, locales e institucionales pueden incorporar al profesorado especializado en sus políticas de desarrollo profesional docente?, ¿qué implicancias educativas tiene el cambio o continuidad de las condiciones laborales luego de la especialización en la retención o movilidad de los docentes nóveles?, ¿qué incidencia educativa tienen los docentes especializados en escuelas públicas con una matrícula alta?, ¿qué modelos teóricos de desarrollo profesional destacados en la literatura internacional permiten comprender los hallazgos de este trabajo? y, en el marco del nuevo sistema de desarrollo profesional docente, ¿cómo pueden ser considerados los hallazgos de este trabajo en las acciones de atracción, retención y formación docente que las reformas intencionan? Sin duda, futuros estudios deberán hacer frente a estas interrogantes e hipótesis que abren los resultados de este trabajo y su contraste con la literatura internacional y nacional en la materia. Asimismo, se espera que los resultados aporten evidencias para el estudio de la pertinencia de las políticas de desarrollo profesional docente en Chile en lo general y de los PPMat en lo particular. 
Finalmente, se puede concluir que los cambios y continuidades de las condiciones laborales en el marco de procesos formación permanente pueden ser considerados factores relevantes en el estudio del desarrollo profesional docente. El principal mensaje de este trabajo es que un docente con especialización profesional y favorables condiciones laborales tenderá no sólo a permanecer en el aula irradiando sus nuevos saberes a sus estudiantes sino que también de forma importante se consolida o moviliza desde establecimientos de carácter privado a público, cumpliendo con ello el ideal democratizador de las reformas educativas en marcha.

\section{REFERENCIAS BIBLIOGRÁFICAS}

Agencia de la calidad (2016). Estadísticas de la educación 2016. Disponible en https://centroestudios. mineduc.cl/wp-content/uploads/sites/100/2017/07/Anuario_2016.pdf

Alain, C., Alain, M., Chabanne, J., Bucheton, D., Demougin, P., Gordon, J., Sellier, M., Udave, J. y Valette, S. (2013). Study on Policy Measures to Improve the Attractiveness of the Teaching Profession in Europe. Unión Europea.

Alvarado, L. (2012). Escuelas: ¿espacios colectivos institucionales de la formación de profesores? Latina. En Arriagada (Ed) Nuevos escenarios y desafíos para los sistemas educativos de América Escuela de Ciencias de la Educación. Cap. 5, 125-142. México.

Amestica, J., Miranda, C., Medina, J., Abarca, R., Medina, J. y Pinto, E. (2018). Significados sobre las diferencias individuales de los estudiantes construidos por profesores en un programa de formación continua: un acercamiento desde la teoría fundamentada. Investigación Cualitativa en Educación 1, 782-791. https://proceedings.ciaiq.org/index.php/ciaiq2018/article/ download/1707/1660/

Ávalos, B. y Matus, C. (2010). La Formación Docente en Chile Desde una Óptica Internacional. Informe Nacional del Estudio Internacional IEA TEDS-M. Santiago, Chile: Ministerio de Educación.

Ávalos, B. y Valenzuela, J. P. (2016). Education for all and attrition retention of new teachers A trajectory study in Chile. International Journal of Educational Development 49, 279-290.

Barría, P., Miranda, C., Toledo, M. y Guajardo, G. (2017). Significados docentes sobre violencias en las relaciones laborales en la escuela. Foro Educacional 29, 13-34. http://ediciones.ucsh.cl/ojs/ index.php/ForoEducacional/article/view/780

Beca, C. (2006). Los programas de formación en servicio del CPEIP. Documento de Trabajo: Santiago: MINEDUC.

Beyer, A., Miranda, C. y Arancibia, M. (2018). Análisis de las concepciones de docentes de primaria que cursan postítulos en Matemáticas: aspectos pedagógicos, disciplinares y epistemológicos. En Torres, B. (Comp) Educación, escuela y profesorado: aportes desde el Consejo de Decanos de Facultades de Educación del Consejo de Rectores de las universidades chilenas (CONFAUCE), 151-172. Concepción: Universidad de Concepción.

Blanco, R. (2013). Formación continua en la comunidad iberoamericana. Ponencias del Seminario Internacional. Formación continua y desarrollo profesional docente. Organización de los Estados Americanos para la Educación, la Ciencia y la Cultura. Santiago, Chile.

Catena, A., Ramos, M. y Trujillo, H. (2003). Análisis multivariado. Un manual para investigadores. México: Biblioteca Nueva.

Centro de Políticas Públicas UC (2012). Propuestas para una carrera docente. Santiago: Elige Educar-PUC.

Cisternas, T. (2016). Profesores principiantes de Educación Básica: dificultades de la enseñanza en contextos escolares diversos. Revista Estudios Pedagógicos, 42(4),135-151. 
Estudios Pedagógicos XLVII N ${ }^{\circ}$ 3: 7-25, 2021

CONDICIONES LABORALES DE PROFESORES EGRESADOS DEL PROGRAMA DE POSTÍTULO DE MENCIÓN EN

MATEMÁTICA PARA SEGUNDO CICLO BÁSICO

Fern, J. (2017). University could do more to stop the exodus from state school teaching. The Guardian. Retrieved from https://www.theguardian.com/teacher-network/2017/aug/16/teacher-rententionuniversitieshelp-mentoring-nqts

Geiger, T. y Pivovarova, M. (2018). The effects of working conditions on teacher retention. Teachers and Teaching, 24(6), 604-625. Doi: 10.1080/13540602.2018.1457524

Goldhaber, D., Krieg, J. y Theobald, R. (2016). Does the match matter? Exploring whether student teaching experiences affect teacher effectiveness and attrition (Working Paper 149). Washington: National Center for Analysis of Longitudinal Data in Education Research.

González, I. (2020). Análisis de las trayectorias profesionales de docentes de Educación Básica que se especializan en Matemática y Ciencias de la Región Metropolitana. Tesis de Grado de Magíster: Santiago: Universidad de Chile.

Hancock, C. B. y Scherff, L. (2010). Who will stay and who will leave? Predicting secondary English teacher attrition risk. Journal of Teacher Education, 61(4), 328-338.

Ingersoll, R. y Strong, M. (2011). The impact of induction and mentoring programs for beginning teachers: A critical review of the research. Review of Educational Research, 81(2), 201-233.

Ladd, E. (2018). Teachers' Perceptions of Their Working Conditions: How Predictive of Planned and Actual Teacher Movement? Educational Evaluation and Policy Analysis, 33(2), 235-261.

Lankford, H., Loeb, S. y Wyckoff, J. (2002). Teacher sorting and the plight of urban schools. A descriptive analysis. Educational Evaluation and Policy Analysis, 24(1), 37-62.

López, I. (2015). Retiro Temprano de Profesores del Sistema Educativo de Chile. Tesis de Grado de Magíster: Santiago: Universidad de Chile.

Martínez, D. (2001). Abriendo el presente de una modernidad inconclusa: treinta años de estudios del trabajo docente. Red Latinoamericana de Estudios sobre Trabajo Docente.

Marcelo, C. (2009). Los comienzos en la docencia: Un profesorado con buenos principios. Revista de Curriculum y formación del profesorado, 13(1), 1-25.

Meckes, L. y M. Bascopé (2010). Distribución inequitativa de los nuevos profesores mejor preparados: Características de origen y destino laboral de los egresados de pedagogía básica. Ponencia en Primer congreso interdisciplinario de investigación en educación. Santiago: Universidad de Chile.

MIDE UC. (2009). Resultados de la Prueba SEPA. Santiago: Centro Mide UC.

MINEDUC (2017). Ley $N^{\circ} 20.903$ de Carrera Docente. Santiago: MINEDUC.

Miranda, Ch., Rivera, P., Arancibia, M. y Osses, S. (2012). ¿Qué hace a la formación permanente de profesores eficaz? Factores que inciden en su impacto. Revista Estudios Pedagógicos, 36(2), 135-151.

Monarca, H. y Manso, J. (2015). Desarrollo Profesional Docente en el discurso de los Organismos Internacionales. Revista Española de Educación Comparada 26, 171-189. Doi: 10.5944/ reec.26.2015.14775.

Mourshed, M., Krawitz, M. y Dorn, E. (2015). How to improve student educational outcomes: New insights from data analytics. Recuperado en https://www.mckinsey.com/industries/public-andsocial-sector/our-insights/how-to-improve-student-educational-outcomes-new-insights-fromdata-analytics

OCDE (2016). Education at a Glance 2016. Paris: OECD. Recuperado en https://doi.org/10.1787/ eag-2016-en

. (2017). Education in Chile, Reviews of National Policies for Education, Paris: OECD. Recuperado en http://dx.doi.org/10.1787/9789264284425-en

. (2018). Education at a Glance 2018, Paris: OECD. Recuperado en http://dx.doi.org/10.1787/ eag-2018-en

. (2019). TALIS 2018 Results (Volume I): Teachers and School Leaders as Lifelong Learners, Paris: OECD. Recuperado en https://doi.org/10.1787/1d0bc92a-en

Puga, I., Polanco, D. y Corvalán, D. (2015). Segregación de la formación y carrera docente y su rol en 
la reproducción social de la desigualdad. Revista Calidad en la educación 43. Doi: http://dx.doi. org/10.4067/S0718-45652015000200003

Quintero, J., Miranda, C. y Rivera, P. (2018). Tendencias de investigación en formación permanente de profesores: estado del arte e interpretación de actores clave. Revista Actualidades Investigativas en Educación 18(2), 1-29. https://doi.org/10.15517/aie.v18i2.33174

Ralph, S., Miranda, C. y García, C. (2019). Modelo de seguimiento basado en TIC de la formación continua de profesores. Revista Runae, 2, 66-88. https://revistas.unae.edu.ec/index.php/runae/ article/view/186

Rivera, P. y Miranda, C. (2009). Perfil profesional y productividad científica del Formador de Formadores: un análisis con perspectiva de género. En Arriagada, M., Ortiz, A. y Browne, R. (Comp.) Comunicación y Género, 107-134. Sevilla: Arcibel Editores.

Salinas, S. (2010). Necesidades de formación permanente de los profesores de segundo ciclo básico participantes en el postítulo de matemática. Tesis de grado Magíster. Valdivia: Universidad Austral de Chile.

Stake, R. (2006). Evaluación comprensiva y evaluación basada en estándares. Madrid: Editorial Grao.

Strauss, A. y Corbin, J. (2002). Bases de la investigación cualitativa. Técnicas y procedimientos para desarrollar la Teoría Fundamentada. Medellín: Universidad de Antioquia.

Sutcher, L., Darling-Hammond, L. y Carver-Thomas, D. (2016). A coming crisis in teaching? Teacher supply, demand, and shortages in the U.S. Palo Alto: Learning Policy Institute. Recuperado en https://learningpolicyinstitute.org/product/coming-crisis-teaching

Terigi, F. (2012). Los saberes de los docentes: Formación, elaboración en la experiencia e investigación. Documento básico, Buenos Aires: Santillana.

Troncoso, A. (2003). Breve historia de la formación de profesores en Chile. En Alvarado L. (Ed.) Formación de profesores en América Latina, 97-124. Bogotá: Antropos.

UNESCO-OREALC (2012). Informe Regional De Monitoreo Del Progreso Hacia Una Educación De Calidad Para Todos En América Latina Y El Caribe. Santiago: Unesco. 
